\title{
THE SYNDROME OF THE SUSPENDED HEART
}

\author{
BY \\ WILLIAM EVANS AND H. G. LLOYD-THOMAS \\ From the Cardiac Department of the London Hospital
}

Received February 27, 1956

When the electrocardiogram of occasional patients in whom a description of pain in the chest did not tally with that customarily identified with cardiac pain was examined, the finding of S-T depression caused surprise and confused the diagnosis. In order to discover the reason for this cardiographic irregularity we assembled for special study 13 patients who possessed such an abnormal tracing which had been recorded in the recumbent position.

A peculiar stance of the heart within the thorax was found common to each case, and in this paper we describe circumstances, the recognition of which will prevent a wrong interpretation of the electrocardiogram obtained in a group of subjects with chest pain.

\section{The CASES Described}

Of the 13 patients, eight were men and five women. Their ages varied from 18 to 68 years; there were eight between 18 and 30, three between 30 and 60, and two over 65 years. All of them were of average height; eight were of slender physical build, four of medium build, and one was stout. Scoliosis was slight in one and moderately severe in another. None showed depression of the sternum and none had emphysema.

Chest pain was a presenting symptom in nine patients and in seven of these it was the only complaint; in the other two it was accompanied by palpitation in one and by loss of weight in the other. Three patients complained of palpitation only, but in none was this caused by paroxysmal tachycardia. The thirteenth was referred for examination as a possible case of mitral stenosis, but this could not be confirmed and the heart was judged to be healthy. In six of the nine patients with chest pain it was felt on the left side and usually below the breast. In the other three the pain was in the region of the right breast in one, behind the sternum in one, and across the chest in the third. The duration of the pain varied from momentary stabs to continuous pain that persisted for a whole week. In three the pain was described as sharp; in five it was an ache, and in one it was likened to a tight band round the chest. Deep inbreathing increased the pain in two, movement of the right arm induced it in one patient, while walking caused it occasionally in another.

Routine clinical examination failed to show evidence of cardiovascular disease in any of the 13 cases, and the blood pressure was never raised. A soft systolic murmur heard to the left of the sternum in two instances was considered to be innocent.

The Electrocardiogram. In each of the 13 cases the electrocardiogram (Fig. 1) showed a design that differed from the accepted normal tracing in a consistent way. Thus, the $\mathrm{T}$ wave was inverted and/or the S-T segment was depressed in lead II and to a greater extent in leads III and IIIR (lead III recorded during deep inspiration). The $\mathrm{T}$ was also low in CR7 in seven cases and the $\mathrm{S}-\mathrm{T}$ segment depressed in another four; in only two was the curve natural in this lead. In two instances there was also depression of the S-T in CR4, and in one of them (Case 6), with severe scoliosis, this change was a noticeable feature.

The $\mathbf{P}$ wave was natural and if bifid it was never tall. The $\mathbf{P}-\mathbf{R}$ segment was frequently depressed, particularly in leads that showed S-T depression. None showed pathological Q waves. 


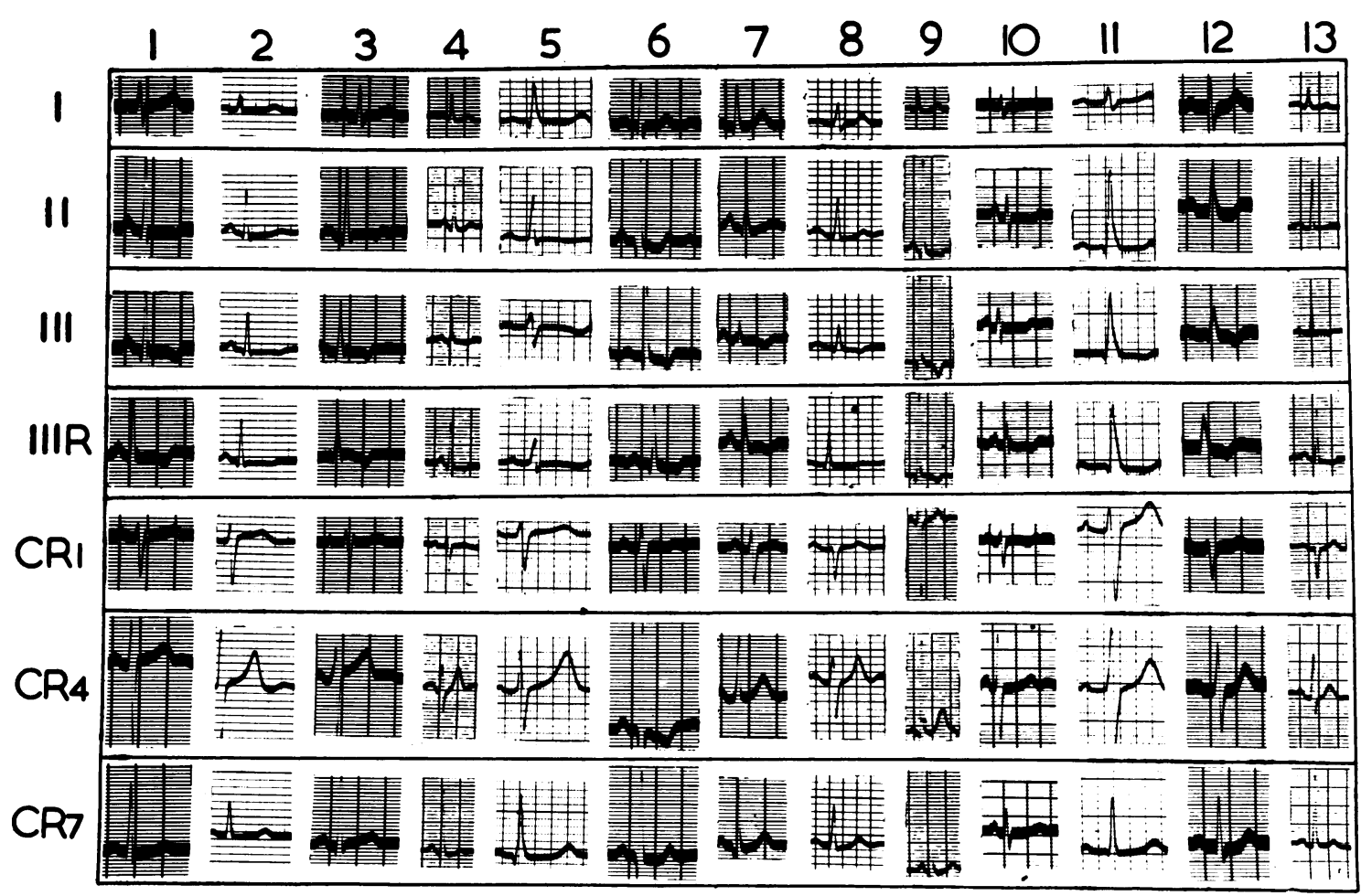

FIG. 1.-The electrocardiogram in 13 patients who showed the syndrome of the suspended heart. The figures above indicate the case numbers.

In lead I the voltage was rather low in seven cases. The $\mathrm{R}$ wave in limb leads was tallest in II and in 10 cases it was taller in III than in I. The S wave in CR1 was always larger than the $R$ wave. In lead I, S was absent in five, small in six, and moderately deep in two: in CR7, S was absent in eight, and small in five cases. The unipolar limb leads supplied no added information. Thus, aVR and aVL were within normal limits, the S-T being depressed in aVF and aVFR (aVF recorded during deep inspiration). The vectorcardiogram when taken did not demonstrate the deformity as clearly as, nor help to explain its mechanism to better advantage than, did the orthodox electrocardiogram.

The tracing repeated after strenuous physical exercise did not show in a single instance any accentuation of the deformity, giving a so-called positive reaction. This test proved valuable in differentiating this innocent tracing from that obtained in cardiac infarction, so placed in the postero-inferior aspect of the heart, as to produce a comparable cardiogram. Thus, strenuous exercise accentuated the deformity in the pathological tracing, giving a positive reaction (Fig. 2).

Radiology of the Heart. The heart was never enlarged. Once it was displaced some distance to the left because of scoliosis. In the other cases it occupied a natural position in the anterior view, and during quiet respiration the customary cardiophrenic relationship appeared to be preserved. On deep inspiration, however, the heart and diaphragm parted to an unusual distance reducing the obtuseness of the left cardiophrenic angle, and on the right side uncovering the inferior vena cava. In the left oblique view the appearance was more arresting, so that the heart, lifted from its diaphragmatic platform, appeared to be suspended in mid-thorax from its vascular pedicle above, and anchored centrally by the inferior vena cava below. Similarly, in the right oblique view the cardio-diaphragmatic separation assumed a characteristic appearance with the slanting course 
FIG. 2.-The resting electrocardiogram (R) from a patient with cardiac infarction showing low $T$ waves in leads II and CR7 and S-T depression in leads III and IIIR. These changes became exaggerated on exercise (E) and the S-T was particularly depressed in leads I, II and CR7.
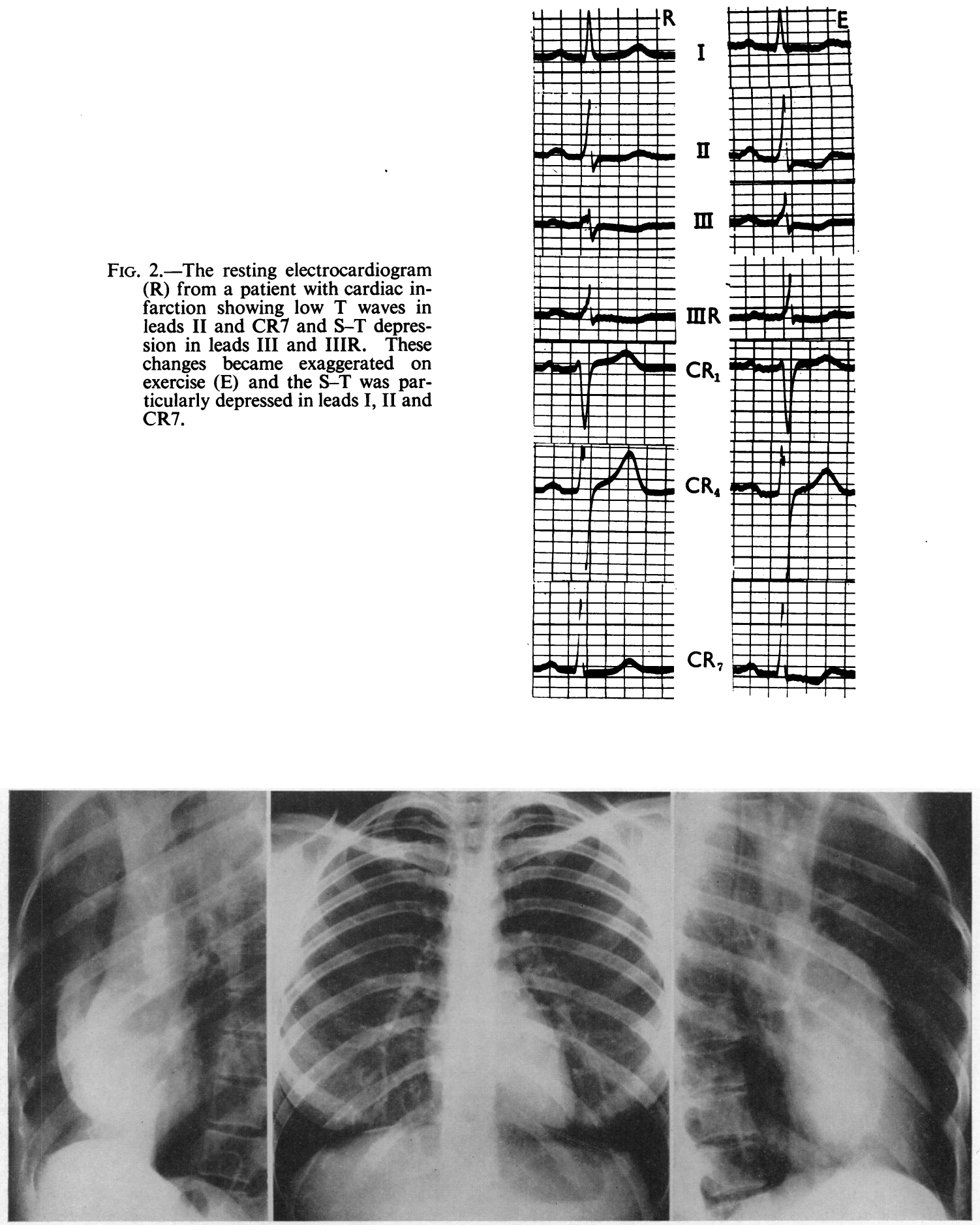

FIG. 3.-Teleradiogram (left oblique, anterior, and right oblique views) from a healthy subject showing the suspended heart. The inferior vena cava, in the oblique views, is exposed in its course from the diaphragm below to the elevated heart above. 


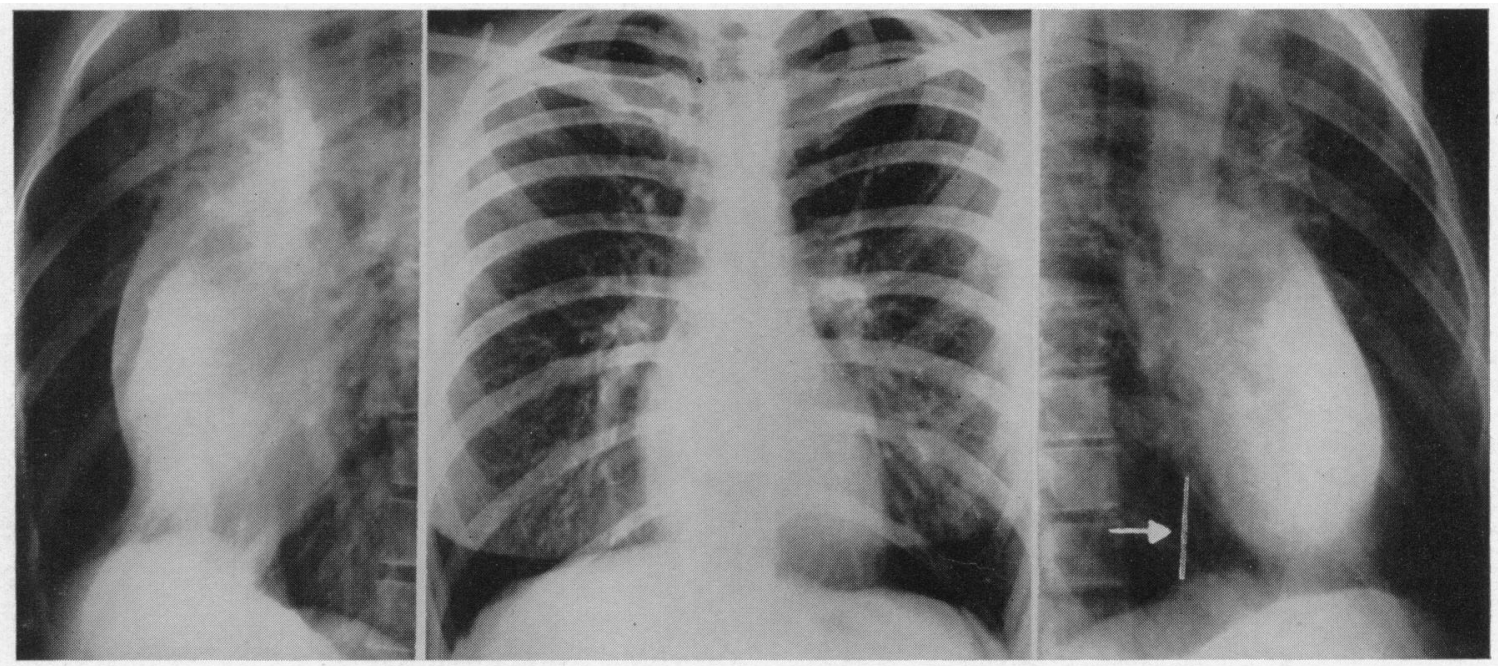

Fig. 4.-Teleradiogram (left oblique anterior, and right oblique, views) from a healthy subject showing the suspended heart. The inferior vena cava, in the oblique views, is exposed in its course from the diaphragm below to the elevated heart above; the arrow points to the line that indicates the vein's right border.

of the inferior vena cava obviously displayed (Fig. 3 and 4). There was no evidence of emphysema in any of the cases.

In a few patients in whom casual radiological examination suggested the characteristic appearance we have just described, the electrocardiogram was a normal tracing; a more critical radiological survey in such patients showed that although the heart appeared unusually elevated in relation to the diaphragm, a great part of the heart remained in contact with the diaphragmatic surface even during very deep inbreathing.

\section{DisCUSSION}

Changes in the electrocardiogram that take place as the result of an alteration in the position of the heart within the chest, and the manner in which its electrical potential is consequently readjusted, have often been described. Herrmann and Wilson (1922) concluded that the form of the natural electrocardiogram was not determined by the relative weight of the two ventricles, but depended chiefly upon the position of the heart and upon the arrangement of the ventricular conducting system; sometimes the one and sometimes the other of these factors exerted the greater influence. Katz and Robinow (1936) thought that the electrical field of the body was modified in accordance with the particular region of the heart that was in contact with the anterior chest wall, with the diaphragm, and with the posterior paravertebral muscle mass, which acted as good electrical conductors compared with the lungs which were poor conductors. Sigler (1938), writing on the changes in the cardiogram that took place in 100 cases when they assumed the standing and sitting posture from the recumbent, reported that in some instances the change was so gross as to cause the tracing to lose its identity from the one recorded in the other posture. Such changes did not conform to the theory based on Einthoven's triangle, for the shift in the electrical axis was not always the same as that predicted from the shift of the anatomical axis. Sigler concluded that the phenomenon was best explained by the change in contact of the adjacent conducting media with different portions of the heart resulting from alteration of body posture and that this produced a variation in conduction. Scherf and Weissberg (1941) from their observations on the effect of posture on the $\mathrm{T}$ wave in lead III, also concluded that these cardiographic changes followed an alteration in the contact taking place between the heart and the neighbouring tissues. 
Writing on the electrocardiogram in its relation to the radiological configuration of the heart, Master (1942) described the long narrow heart in subjects with an asthenic habitus and with symptoms associated with neurocirculatory asthenia, and mentioned that in the past the terms hypoplastic, asthenic, drop, and emphysema heart, had been applied to it. In such cases where the diaphragm was depressed and the heart appeared elongated and vertical, the cardiogram was characterized by a low amplitude of the $\mathbf{P}$ and $\mathbf{R}$ waves in lead I and a high amplitude in leads II and III. Katz (1946) described the same tracing and stated that there was no unusual deviation in the S-T segment. A code of normality for the behaviour of the curve in lead III when repeated during deep inspiration (lead IIIR) was drawn up by Evans (1951). Thus, the P, R and T waves are expected to move upwards significantly, and without depression of the S-T segment, on deep inspiration. This is true for the large majority of healthy subjects, but in order that it might apply unexceptionally, it is necessary at cardioscopy to witness during the inspiratory phase a descent of the heart in unison with the diaphragm. Whenever this natural cardiodiaphragmatic relationship is maintained, depression of the T wave and of the S-T segment in IIIR confirms the existence of heart disease, provided also that it is not a digitalis effect.

Another distinctive electrocardiogram was described by Ylvisaker and Kirkland (1940) in a group of healthy young subjects in whom changes in the T wave in leads II and III, that could not be regarded as those accepted as variations of the normal, disappeared when the reclining position was assumed. In some of the cases a change into the upright position caused the T wave in lead II to become diphasic or iso-electric and in lead III to become inverted; a return to the recumbent position invariably reinstated the $\mathrm{T}$ wave in lead II to a normal configuration and amplitude, but a change in lead III was less constant and not so marked. Holzmann (1952) described the same cardiogram in subjects of subnormal body weight and with an asthenic habitus, where the $\mathbf{P}$ and $\mathbf{R}$ waves are tall in leads II and III and where the S-T segment and T wave are depressed predominantly in leads II and III and in chest leads over the left heart. He stated that such a tracing was obtained with the subject standing upright or fixed to a steeply inclined plane, and that the changes disappeared immediately on a return to the horizontal position. Goldberger (1953) proposed a remarkable explanation for such postural changes in the $T$ wave; he postulated that in the upright position, when blood is pooled in the lower extremities, sympathetic stimulation following the transient drop in blood pressure causes a decrease in blood potassium and in turn reduces the amplitude of the $\mathrm{T}$ wave and depresses the $\mathrm{S}-\mathrm{T}$ segment. If this were the explanation it is difficult to understand why the same changes should not be visible in the $T$ wave in some of the other leads.

In this paper we describe an electrocardiogram that exhibits its special characteristics in the reclining position so that any postural manœuvre does not change its design, and for that reason cannot help its identification from the pathological variety met with in cardiac infarction. A somewhat similar tracing has been published by Master (1942) for his Fig. 24, taken from a youth aged 21 in whom radiological examination had shown a long and narrow heart, shows a cardiogram where the T wave was inverted in leads II and III. Master considered that clockwise rotation of the heart rather than impairment of the heart muscle had caused these changes. The fact that postural manœuvre fails to change this cardiogram into one of normal pattern, thereby establishing its innocent nature, creates a difficulty in telling it from the tracing that confirms the presence of cardiac infarction. In the pathological cardiogram, however, recorded in a patient the subject of cardiac pain, the existing S-T depression is exaggerated following strenuous physical exercise. In the innocent cardiogram, although the changes at rest were similar to those in the pathological tracing, they were not exaggerated by exercise; and at the same time specific radiological appearances connected with the peculiar position of the heart were found to be present.

We examined the electrocardiogram in 33 patients with pulmonary emphysema in whom radiology of the chest had shown a low and rather immobile diaphragm and a centrally placed heart that looked small and slender in all but three cases where it was enlarged. In 27 of these the electrocardiogram was physiological; in the three with cardiac enlargement the tracing showed 
right heart preponderance; in the remaining three the electrocardiogram was similar to the one we describe in this paper, but the S-T segment was not depressed in CR7.

The separation of the suspended heart from its close contiguity with its surrounding structures, particularly the diaphragm, disturbs the anatomical medium that customarily transmits the varying electrical potential generated by cardiac contraction, and this we consider gives rise to changes associated with this characteristic cardiogram.

\section{Summary AND CONCLUSIONS}

In the absence of digitalis therapy, depression of the S-T segment in the electrocardiogram recorded in the reclining position is usually accepted as evidence of myocardial injury from coronary arterial disease or as a sign of ventricular hypertrophy. In a group of patients, however, we found it inappropriate on clinical grounds to apply so sinister a meaning to this cardiographic sign, and for this reason we assembled 13 such cases for special investigation.

Each tracing showed depression of the S-T segment in leads III and IIIR (lead III recorded during deep inspiration), and lesser depression in leads II and CR7, and it remained materially unaltered by a change to the upright posture. The cardiogram resembled that found in cardiac infarction involving the lateral and postero-inferior portion of the left ventricle. The innocent tracing that we describe here, differed however, from the pathological kind in two ways, namely that strenuous physical exercise failed to increase the deformity and that it was associated with characteristic radiological changes.

Chest pain had brought the heart under suspicion in 9 of the 13 patients, although almost invariably, either in regard to its character, distribution, or its response to exercise and rest, it failed to conform to the tenets customarily applied to true cardiac pain. Although two patients were over 65 the fact that eight were under 30 years of age was foremost in suggesting that cardiac infarction had not caused the cardiographic irregularity which it so closely resembled.

Radiological examination of the heart disclosed an appearance that proved to be common to all cases and indicated a peculiar stance of the heart as the explanation for the abnormal electrocardiogram. In the anterior view casual examination often discovered nothing more than a naturally placed heart, but during deep inspiration the left cardiophrenic junction moved noticeably towards the middle and the angle narrowed correspondingly; on the right side the cleavage between the right atrium and the diaphragm laid bare the inferior vena cava as it traversed this gulf. During inspiration in the right oblique, the heart aloft and the diaphragm below separated widely, and again brought the inferior vena cava, which coursed slantwise across the gap, into great prominence. Similarly in the left oblique view the heart during inspiration was lifted some way above the depressed diaphragmatic platform to which it appeared anchored centrally by the inferior vena cava.

The recognition of these characteristic electrocardiographic and cardioscopic findings, featuring the syndrome of the suspended heart, should avoid the erroneous diagnosis of coronary arterial disease in subjects in whom chest pain is associated with a depression of the S-T segment in the electrocardiogram.

\section{REFERENCES}

Evans, W. (1951). Brit. Heart J., 13, 457.

Goldberger, E. (1953). Unipolar Lead Electrocardiography and Vectorcardiography. Kimpton, London.

Herrmann, G. R., and Wilson, F. N. (1922). Heart, 9, 91.

Holzmann, M. (1952). Clinical Electrocardiography. Staples Press, London.

Katz, L. N. (1946). Electrocardiography. 2nd ed., Kimpton, London.

- and Robinow, M. (1936). Amer. J. med. Sci., $192,556$.

Master, A. M. (1942). The Electrocardiogram and X-Ray Configuration of the Heart. 2nd ed., Kimpton, London.

Scherf, D., and Weissberg, J. (1941). Amer. J. med. Sci., 201, 693.

Sigler, L. B. (1938). Amer. Heart J., 15, 146.

Ylvisaker, L. S., and Kirkland, H. B. (1940). Amer. Heart J., 20, 592. 